\title{
Iterative Receiver Signal Processing for Joint Mitigation of Transmitter and Receiver Phase Noise in OFDM-Based Cognitive Radio Link
}

\author{
Ville Syrjälä and Mikko Valkama \\ Department of Communications Engineering \\ Tampere University of Technology \\ P.O. Box 553, 33101 Tampere, Finland \\ ville.syrjala@tut.fi and mikko.e.valkama@tut.fi
}

\begin{abstract}
In opportunistic spectrum access and cognitive radio, the device is assumed to be operating over a very wide range of radio frequencies (RF). This implies that severe implementation imperfections can take place in the RF modules of the devices. One such imperfection is oscillator phase noise. In this article, we study the impact and DSPbased mitigation of oscillator phase noise in OFDM-based cognitive radio link. The paper proposes an iterative receiverside DSP algorithm for joint transmitter and receiver phase noise mitigation. In the algorithm, the received signal is first detected, and the detection results combined with channel state estimate are used to form an estimate of the time-varying phase noise process. This phase noise estimate is then used to suppress the phase noise effects from the original received signal. Simulation results show that the proposed algorithm gives significant performance improvement over the existing phase noise mitigation algorithms.
\end{abstract}

Index Terms-Cognitive radio, OFDM, phase noise, mitigation, digital signal processing, Dirty-RF

\section{INTRODUCTION}

$\mathrm{O}$ RHOGONAL frequency division multiplexing (OFDM) is a widely-applied way to convey information in spectrally efficient manner in digital radio communications. It is also the basis in many emerging cognitive radio developments, offering possibilities to utilize multiple, and possibly scattered, frequency slices for secondary radio communications through

This work was supported by Tampere University of Technology graduate school, Jenny and Antti Wihuri Foundation, Ulla Tuominen Foundation, HPY Research Foundation, Academy of Finland (under the project "DigitallyEnhanced RF for Cognitive Radio Devices"), the Finnish Funding Agency for Technology and Innovation (Tekes; under the projects "Enabling Methods for Dynamic Spectrum Access and Cognitive Radio" and "Reconfigurable Antenna-based Enhancement of Dynamic Spectrum Access"), the Austrian Competence Center in Mechatronics (ACCM, Austria). properly assigned subcarrier allocations [1]. However, OFDM as such is also very sensitive to many transceiver/RF implementation imperfections, like I/Q imbalance, phase noise and transmitter nonlinearities [2], [3]. Such RF imperfection aspects are emphasized even more in dynamic spectrum access and cognitive radio (CR) where the used radios, when understood at large scale, should operate over extremely wide bandwidths, covering several decades of spectrum $(0.01 \ldots 10 \mathrm{GHz})$ as a whole, and be able to sense and communicate under extreme dynamic range conditions in the order of 50-100 dB. This has been recently acknowledged by the leading researchers in the field, e.g., in [4].

In this article, we focus particularly on oscillator phase noise and its effects in OFDM-based radio communications and cognitive radio. In the state-of-the-art literature, the phase noise mitigation in OFDM systems has already been widely studied. Advanced phase noise mitigation techniques were already discussed, e.g., in [5], [6], [7], [8], [9], [10] and [11]. To the authors' best knowledge, even though being computationally demanding, the phase noise mitigation technique in [11] is currently the best performing technique available. This paper proposes significant improvements to the compensation structure. The compensation structure proposed in this paper is built to improve the performance mainly when a challenging communications channel is present, whereas the previous algorithm was built originally from the no-channel perspective. Also, the algorithm in [11] did not exploit the cyclic prefix. However, in the proposed algorithm, the structure is changed so that the cyclic prefix can be exploited in the phase noise estimation process. As illustrated in this article, the proposed method clearly outperforms all the existing state-of-the-art methods, including the method in [11].

This paper is structured as follows. The Section II gives shortly the phase noise and OFDM link models. The actual phase noise mitigation algorithm is presented in Section III. In Section IV, the performance of the proposed phase noise mitigation algorithm is compared to that of the state-of-the-art 
techniques, and finally Section $\mathrm{V}$ concludes the work.

\section{Phase NoISE AND OFDM Link Modelling}

This section shortly describes the used phase noise model. It also gives OFDM link model used in the simulations and in the derivation of the proposed phase noise mitigation algorithm.

\section{A. Phase Noise Model}

The phase noise model used in this paper is very simple free-running oscillator (FRO) model very often used in the literature [12]. Previous studies have shown that the FRO model is very demanding for phase noise mitigation algorithms [6], [9], [10], [11]. It is thus sufficient for the performance evaluation of such algorithms. If the studied algorithms are capable of mitigating the phase noise of the wandering nature, they are able to mitigate, e.g., phase-locked-loop (PLL) type phase-noise with ease. Therefore, in this paper, the results with PLL oscillator are omitted, and only the more challenging case of FRO is considered for the sake of compactness.

Sampled FRO phase noise sequence is easily generated by cumulatively summing white Gaussian noise samples of certain variance [12]. This kind of a process is called Wiener process or Brownian motion and the $l$ th sample of such process can be written with the help of the standard Brownian motion as

$$
\phi_{l}=\sqrt{c} B\left(l T_{s}\right)
$$

where $B(\cdot)$ denotes the standard Brownian motion, $c$ is the variance of the cumulatively summed white Gaussian noise, namely diffusion rate, and $T_{s}$ is the sampling interval. For the standard Brownian motion, it is known that the spectrum has the well-known Lorentzian shape [12]. From this fact we are able to derive the one-sided 3-dB bandwidth of the process in (1) as

$$
\beta=\frac{c}{4 \pi}
$$

and thus the whole process can be written with the help of $\beta$ as

$$
\phi_{l}=\sqrt{4 \pi \beta} B\left(l T_{s}\right)=\sqrt{4 \pi \beta T_{s}} B(l) .
$$

Here, since $l \in \mathbb{N}$, process $B(l)$ is a cumulative sum of standard normal distributed noise. The whole process is characterized with a single parameter $\beta$ (in addition of the sampling interval naturally.)

\section{B. OFDM Link Model under Phase Noise}

OFDM symbol is generated by inverse discrete Fourier transforming block of $N$ modulated symbols. The $n$th sample of the $m$ th OFDM symbol in the resulting OFDM signal with $N$ subcarriers can then be written as

$$
x_{n}(m)=\frac{1}{\sqrt{N}} \sum_{k=0}^{N-1} X_{k}(m) e^{j 2 \pi k n / N},
$$

where $X_{k}(m)$ for $k=0,1, \ldots, N-1$ are the subcarrier modulated symbols. In practice, also cyclic prefix is used in OFDM signal. This is simply done by sending $G$ last samples of each OFDM symbol before the actual symbol is sent. When the signal with cyclic prefix goes through a channel whose maximum delay spread is shorter than the cyclic prefix, the $m$ th OFDM symbol at the receiver after cyclic prefix removal can be written in vector form as

$$
\mathbf{r}_{m}=\left(\mathbf{h}_{m} * \mathbf{x}_{m}\right)+\mathbf{z}_{m}=\mathbf{H}_{m} \mathbf{x}_{m}+\mathbf{z}_{m},
$$

where $\mathbf{r}_{m}$ is the $N \times 1$ vector of the received samples, and $*$ is circular convolution operator. $\mathbf{h}_{m}$ is $D \times 1$ channel impulse response vector, $\mathbf{x}_{m}$ is $N \times 1$ vector having OFDM symbol samples given in (4) as its elements, $\mathbf{z}_{m}$ is vector of additive white Gaussian noise and $\mathbf{H}_{m}$ is $N \times N$ circulant convolution matrix [13]. This is the model for the OFDM link without any phase noise.

After also phase noise of the upconverting oscillator in the transmitter and the downconverting oscillator in the receiver are taken into account, the received signal can be written as

$$
\mathbf{r}_{m} \approx \operatorname{diag}\left(e^{j \phi_{R, m}}\right) \mathbf{H}_{m} \operatorname{diag}\left(e^{j \phi_{T, m}}\right) \mathbf{x}_{m}+\mathbf{z}_{m} .
$$

Here $\operatorname{diag}(\cdot)$ transforms the input vector to a diagonal matrix, and $\phi_{T, m}$ and $\phi_{R, m}$ are $N \times 1$ vectors of sampled transmitter and receiver phase noises, respectively. The above equation is only an approximation, because the cyclic prefix and the corresponding end part of the OFDM symbol are multiplied with different phase-noise complex-exponentials. Therefore, the cyclic prefix does not precisely work as intended. Then since we also know that $\mathbf{H}_{m}$ is (by definition) circulant matrix, we are able to rewrite (6) as

$$
\mathbf{r}_{m} \approx \operatorname{diag}\left(e^{j \phi_{R, m}}\right) \operatorname{diag}\left(e^{j \phi_{T, m}}\right) \mathbf{H}_{m} \mathbf{x}_{m}+\mathbf{z}_{m}
$$

In this form, we effectively mapped the transmitter phase noise as receiver phase noise, and by writing $\phi_{m}=\phi_{R, m}+\phi_{T, m}$, we can further simplify (7) into form

$$
\mathbf{r}_{m} \approx \operatorname{diag}\left(e^{j \phi_{m}}\right) \mathbf{H}_{m} \mathbf{x}_{m}+\mathbf{z}_{m} .
$$

From the above model, if taken to frequency domain through FFT (as done e.g. in [6]), it is clear that phase noise causes intercarrier interference (ICI). This is further emphasized if some of the subcarriers, like the neighbouring channel subcarriers, are more powerful than those that our receiver is interested in. This is exactly the scenario in cognitive radio where the available spectral chunks are surrounded in frequency domain by strong primary user signals. This very simple form is used as a basis to derive the phase noise mitigation algorithm. The performance of the derived algorithm is in the end used as justification of the used approximations.

\section{Proposed Phase Noise Mitigation Algorithm}

From the received signal, first the cyclic prefix is removed, and then the signal is OFDM demodulated by discrete Fourier 
transform. After this, the channel is estimated and equalized, and common phase error (CPE) [5], [6] is estimated and removed. Finally, the symbols are detected. At this point, the receiver has done everything that conventional OFDM receiver with CPE mitigation block does to obtain symbol decisions. These operations are also depicted in the overall structure of the algorithm in Fig. 1.

After conventional symbol detection with CPE mitigation, the proposed structure reconstructs the sent time-domain waveform by doing inverse discrete Fourier transform and cyclic prefix addition. This is followed by channel modelling, which is done based on the channel estimate. The signal at this point is an approximate of the received waveform without phase noise, $\hat{\mathbf{r}}_{m, n o P N}^{(C P)}$. Now, when we multiply the received waveform $\mathbf{r}_{m}^{(C P)}$ by the complex conjugate of this phase-noise free estimate of the received waveform, the result is a very crude estimate of the phase noise complex exponential, but with some non-constant amplitude. The resulting signal can be written as

$$
\begin{aligned}
\boldsymbol{\varphi}_{m} & \approx \operatorname{diag}\left(\hat{\mathbf{H}}_{m}^{*} \hat{\mathbf{x}}_{m}^{*}\right)\left[\operatorname{diag}\left(e^{j \boldsymbol{\phi}_{m}}\right) \mathbf{H}_{m} \mathbf{x}_{m}+\mathbf{z}_{m}\right] \\
& \approx \operatorname{diag}\left(\hat{\mathbf{H}}_{m}^{*} \hat{\mathbf{x}}_{m}^{*}\right) \operatorname{diag}\left(e^{j \boldsymbol{\phi}_{m}}\right) \mathbf{H}_{m} \mathbf{x}_{m}+\operatorname{diag}\left(\hat{\mathbf{H}}_{m}^{*} \hat{\mathbf{x}}_{m}^{*}\right) \mathbf{z}_{m} \\
& \approx \operatorname{diag}\left(\left|\hat{\mathbf{H}}_{m, C P \mathrm{E}} \hat{\mathbf{x}}_{m}\right|^{2}\right) e^{j\left(\boldsymbol{\phi}_{m}\right)}+\operatorname{diag}\left(\hat{\mathbf{H}}_{m}^{*} \hat{\mathbf{x}}_{m}^{*}\right) \mathbf{z}_{m}
\end{aligned}
$$

Here, $\hat{\mathbf{x}}_{m}$ and $\hat{\mathbf{H}}_{m}$ are the estimates of the sent symbols and the channel convolution matrix, respectively. Superscript $*$ denotes the complex conjugate. As seen from (9), the estimate is very crude as it still has the additive noise component present. Furthermore, each of the sample estimates are multiplied by the corresponding approximate power of the received signal samples without noise.

To greatly improve the estimate of the phase noise complex exponential in (9), the estimate is low-pass filtered. This is a very natural way to improve the estimate since we know that phase noise and its complex exponential are both steep lowpass processes. Therefore also the used filter must be relatively selective low-pass filter. The filter should be designed so that is passes through only few of the centre-most spectral components of the phase noise complex exponential and so that it attenuates the other components heavily because of the noise in them [6], [11]. Prior to the low-pass filtering it is good to scale the signal so that the most reliable sample estimates get more weighted in the filtering process. Fortunately, the scaling has already been done. The sample estimates of the phase noise complex exponential in (9) have indeed already been multiplied by the corresponding approximate powers of the received signal samples without noise, since it is built-in in the multiplication of a signal with its complex conjugate (in [11] the scaling was separately applied, since division operator was used instead of complex conjugate multiplication). This scaling gives more weight to samples that are estimated to have more power at the receiver input, so they are most likely least corrupted by the noise. After the very selective low-pass filter (the filter must be very selective since in (9) the additive

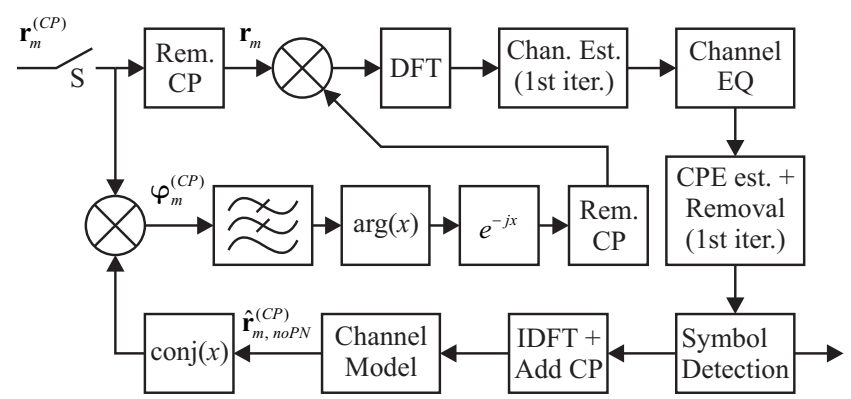

Fig. 1. The proposed phase noise mitigation algorithm. The switch $\mathrm{S}$ remains open until the iterations have been completed, and closes to receive next incoming OFDM symbol. Symbol $x$ always denotes the block input. Blocks $\arg (x)$ and $\operatorname{conj}(x)$ take an argument and a complex conjugate of the input samples, respectively.

noise contributed term dominates the phase-noise complexexponential everywhere else except at very low frequencies), the estimate of the complex exponential is then given by

$$
\operatorname{LPF}\left\{\boldsymbol{\varphi}_{m}\right\} \approx e^{j \phi_{m}}
$$

Even though this is an estimate of the complex exponential, the absolute values of the samples are not unity as they should be. This is why we also take the argument of (10), and then the inverse complex exponential of the result as depicted in Fig. 1. Finally, the received signal without cyclic prefix is multiplied with the inverse complex exponential (from which the cyclic prefix part is also removed) to get rid of the phase noise. The performance of the technique can then be improved by using it iteratively. The complete iterative phase-noise mitigation algorithm is depicted in Fig. 1.

Notice that for notational simplicity, the cyclic prefix is not considered present in the equations. However, as denoted by superscript $(C P)$ in the signals depicted in Fig. 1, the cyclic prefix is indeed present in the corresponding signals in the phase-noise estimation part of the algorithm.

It should also be noted that if the channel estimation is done OFDM-symbol-by-OFDM-symbol, CPE estimation and removal is done during the channel estimation and equalization automatically. In Fig. 1 the blocks are however separate if, e.g. the channel is considered quasistatic and some advanced channel estimation method is used, as e.g. the one proposed in [10]. If desired, the channel estimation can be done only in the first iteration for computational simplicity. However, for improved performance, the structure allows to do the channel estimation again when the amount of phase noise has been lowered by the previous iterations. This again helps channel estimation reliability. The CPE estimation and removal are only done in the first iteration, because the proposed algorithm does not discriminate between CPE and ICI parts of the phase noise. Therefore, in every iteration of the algorithm, CPE and ICI are both mitigated, and hence separate CPE estimation and mitigation parts do not have any practical impact on the quality of the phase noise estimates in the latter iterations.

For the design of the low-pass filter, the considerations given in [11] also apply for the proposed algorithm. This 
means that the used relatively selective, and thus long, digital low-pass filter causes a potential transient problem in the estimate. However, keeping the cyclic prefix present in the algorithm tackles the problem partially.

\section{Simulations ANd PERformance Analysis}

This section gives the used parameters and describes the simulator. It also gives the simulation results and compares the performance of the proposed phase noise mitigation algorithm to the performances of the state-of-the-art phase noise mitigation algorithms reported in [6], [7], [9] and [11]. The simulations are first run for all the reference techniques with perfect channel information. The best performing techniques are then compared in more practical channel estimation cases with imperfect channel information.

\section{A. Parameters and Simulator}

In the simulator we simulate OFDM communications system with 1024 subcarriers. 300 subcarriers on the both sides of the centre subcarrier are active, and the remaining subcarriers are null. The 600 active subcarriers are 16QAM subcarrier modulated. For perfect channel information case, 18 of the active subcarriers are used as pilot subcarriers, i.e., considered known at the receiver. In cases with channel estimation, every ninth subcarrier is considered a pilot, resulting in total of 66 pilot subcarriers. Furthermore, cyclic prefix of 63 samples is present in the system. Assuming $15 \mathrm{kHz}$ subcarrier spacing, this maps to around $10 \mathrm{MHz}$ total waveform bandwidth and 4.2 microsecond cyclic prefix.

The simulator first generates 16QAM symbols and OFDM modulates them. Then cyclic prefix is added and transmitter phase noise is applied. After this, communications channel is modelled. We use extended ITU-R Vehicular A (VEHA) multipath channel [14]. The channel is considered constant during one OFDM symbol, and it is generated independently for all the OFDM symbols, except for the case when advanced channel estimation technique of [10] is considered. For that case, channel is assumed quasistatic for the duration of 12 OFDM symbols. After the channel, at the receiver input, the additive noise is added to get the desired signal-to-noise ratio (SNR). Receiver phase noise is then modelled. At this point, the proposed algorithm is applied, or for the reference techniques, needed operations and algorithms are applied. The parameters of the state-of-the-art algorithms are chosen as in [9] and [11] (optimized empirically for the best estimation quality). For the proposed algorithm, the digital low-pass filter is designed with well-known Remez-algorithm. The order of the filter is 350 with passband width $0 \mathrm{~Hz}$ and normalized stopband edges at 0.04 and 1 . For channel estimation cases, the channel estimation is done only in the first iteration for the proposed technique, and after the CPE mitigation for the reference techniques. Finally, the simulator computes the symbol-error rates (SER) from the detected signals.

Reported 3-dB bandwidth of the phase noise is the 3-dB bandwidth of the total phase noise including the transmitter and receiver phase noises. Both the phase noise processes are independent but with the same diffusion rate.
In the simulations the reference techniques for performance comparisons are denoted by Petrovic, Bittner, LI-TE and Previous for the techniques in [6], [8], [9] and [11], respectively. In Fig. 2, Fig. 3, Fig. 6 and Fig. 7, the techniques are iterated 3 times. In Fig. 4 and Fig. 5, the performance of the proposed technique is compared to the performance of the best performing reference technique (Previous from [11]) from the number of iterations perspective.

\section{A. Simulation Results and Analysis}

The simulation results as a function of received SNR and phase noise 3-dB bandwidth are given in Fig. 2 and Fig. 3, respectively. From the results, we see that the proposed technique gives noticeable performance improvement over the state-of-the-art techniques overall. From Fig. 2 for fixed $\beta$ of $350 \mathrm{~Hz}$, we see that the performance given by the proposed algorithm is very near to the no phase-noise case up until around $25-\mathrm{dB}$ received SNR. After that it starts to floor, but at much lower level than the reference techniques. From Fig. 3 we can see that for fixed received SNR of $24 \mathrm{~dB}$, the proposed

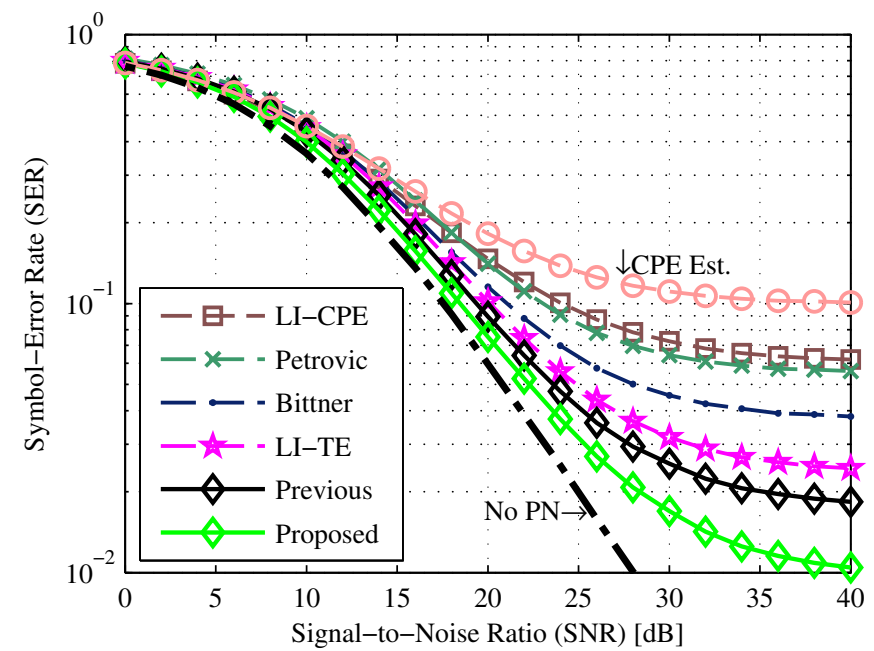

Fig. 2. SER as a function of received SNR. Phase noise 3 -dB bandwidth $(\beta)$ is fixed to $350 \mathrm{~Hz}$

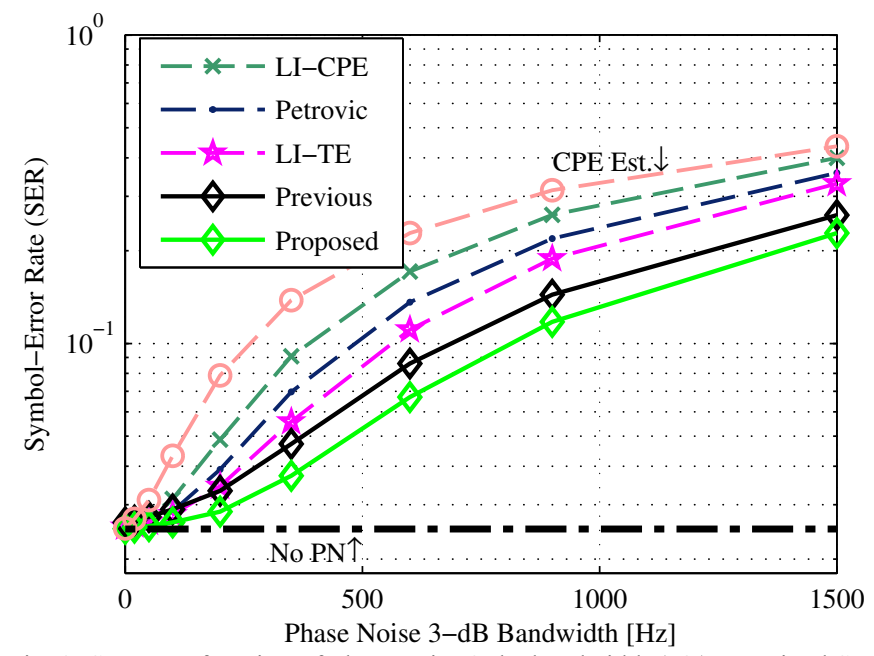

Fig. 3. SER as a function of phase noise $3-\mathrm{dB}$ bandwidth $(\beta)$. Received SNR is fixed to $24 \mathrm{~dB}$. 
algorithm performs very well over the whole studied phase noise 3-dB bandwidth region, and manages to clearly outperform the reference techniques. Altogether, the results also verify the used approximations in earlier signal modelling and in deriving the mitigation algorithm, since the transmission chain in the simulator does not use any approximations.

The simulation results from the amount of iteration perspective are shown in Fig. 4 and Fig. 5. As Fig. 4 shows, already three iterations of the proposed technique clearly outperforms the Previous technique in [11] for fixed $\beta$ of $350 \mathrm{~Hz}$ over the whole studied SNR region. As Fig. 5 depicts, when phase noise gets more dominating, the previous technique gets a little better, but only hardly outperforms the three iterations of the proposed technique with five of its iterations. Overall, the proposed technique can still improve the performance greatly when number of iterations increases.

The simulations were also run for case of additive white Gaussian noise channel. As expected, improvements were seen in the performance compared to the technique of [11]. At SER

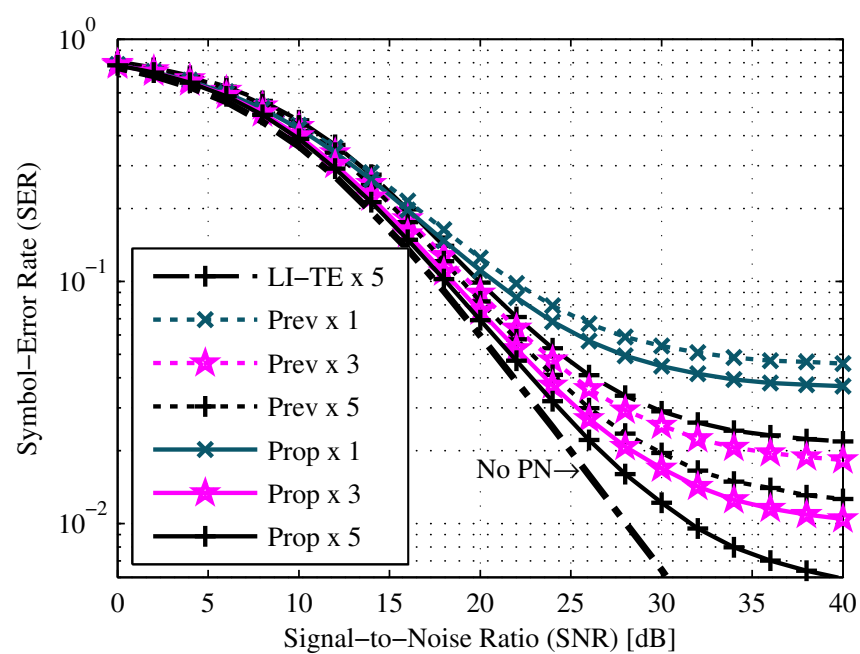

Fig. 4. SER as a functions of received SNR. Phase noise $3-\mathrm{dB}$ bandwidth $(\beta)$ is fixed to $350 \mathrm{~Hz}$.

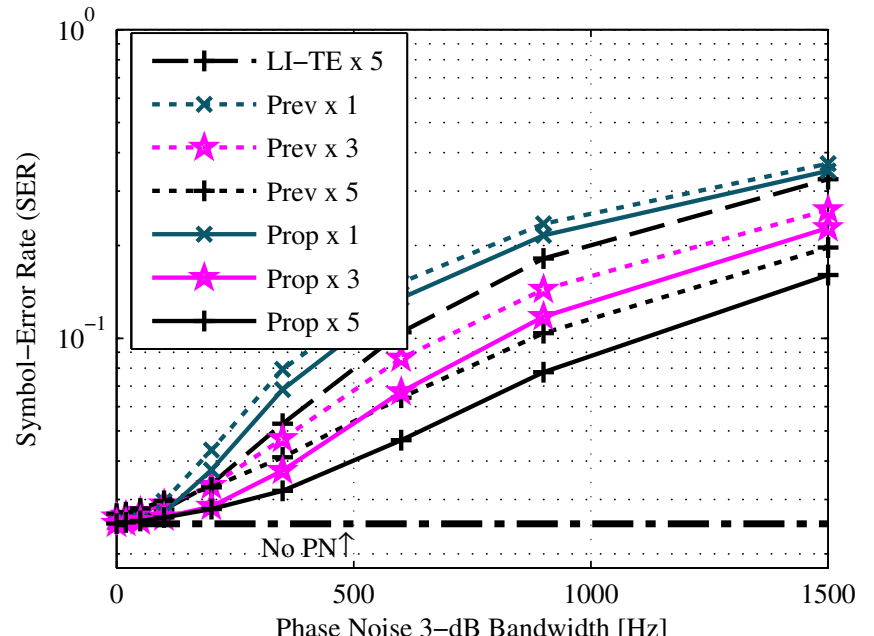

Fig. 5. SER as a function of phase noise $3-\mathrm{dB}$ bandwidth ( $\beta$ ). Received SNR is fixed to $24 \mathrm{~dB}$. of $10^{-2}$, around $1 \mathrm{~dB}$ improvement was got at $350 \mathrm{~Hz}$ phase noise level compared to technique of [11]. Also the SER performance floor was lowered from around $6 \cdot 10^{-3}$ to around $5 \cdot 10^{-3}$ with the same phase noise level. The improvements were gained because the proposed algorithm structure enabled the exploitation of the cyclic prefix in the phase noise estimation process, so the filter transient problem of [11] was partly solved. The performance simulation curves were omitted from this paper for compactness of the presentation. Overall, the performance improvements in additive white Gaussian noise conditions were significant, but clearly smaller than in extended ITU-R Vehicular A multipath channel case.

The simulation results for cases with channel estimation are depicted in Fig. 6 and Fig. 7. In the figures, conventional channel estimation refers to channel estimation done by estimating channel at pilot subcarriers and linearly interpolating the results to get the other channel estimates. The advanced channel estimation refers to the technique proposed in [10]. The results with these channel estimation approaches are compared to the case with perfect channel information at

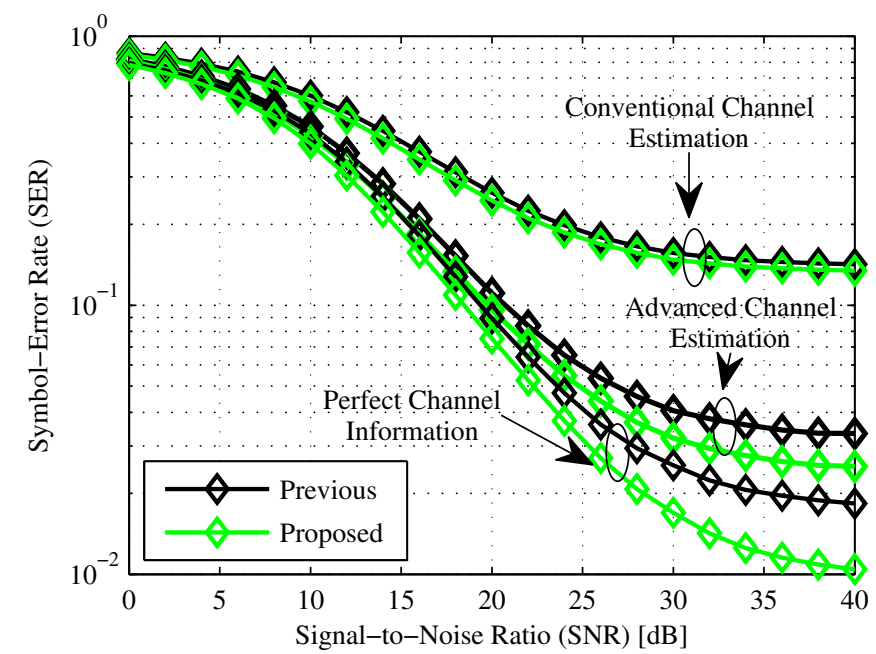

Fig. 6. SER as a functions of received SNR. Phase noise 3-dB bandwidth $(\beta)$ is fixed to $350 \mathrm{~Hz}$.

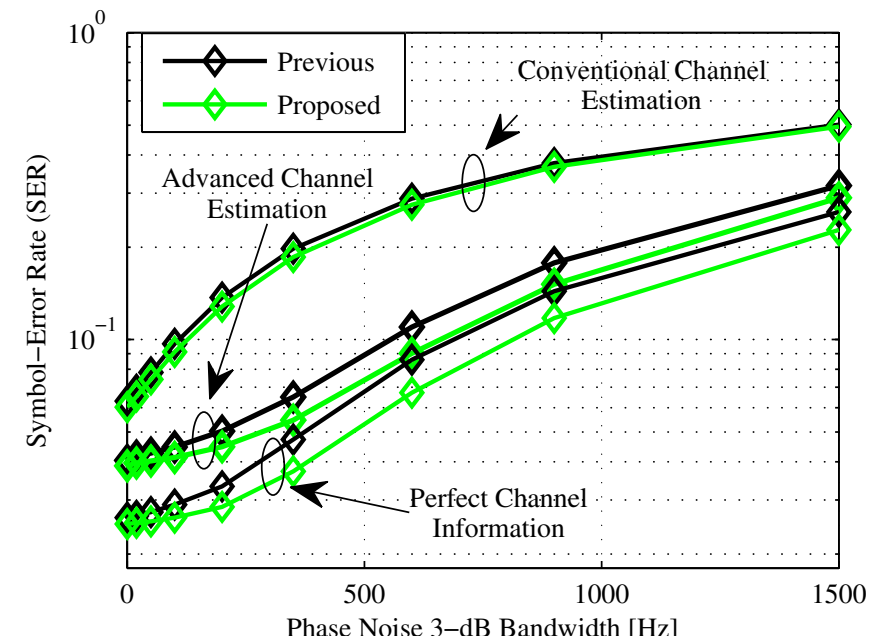

Fig. 7. SER as a function of phase noise $3-\mathrm{dB}$ bandwidth $(\beta)$. Received SNR is fixed to $24 \mathrm{~dB}$. 
the receiver. The results in Fig. 6 and Fig. 7 clearly demonstrate that the proposed technique outperforms the best performing reference technique of [11] also when different channel estimation approaches are used. Already very good performance is achieved with advanced channel estimation compared to the case with perfect channel information.

Overall superior performance of the proposed algorithm when compared to the performance of the algorithm of [11], in case of frequency-selective multipath-channel, is explained by the fact that the phase noise estimation is done before the channel equalization. This effectively means that the noise does not get amplified at some (channel dependent) frequencies in the signal from which the estimation is done (received signal with phase noise, $\mathbf{r}_{m}^{(C P)}$ ), as it does in algorithm of [11]. Of course the channel modelling on the reference signal (reconstructed received signal, $\hat{\mathbf{r}}_{m, n o P N}^{(C P)}$ ) causes possible erroneous subcarriers to be amplified when the channel is strong. However, when channel is strong, also the subcarrier decisions are more likely true. Therefore, actually this amplifies the more probable subcarriers and gives less weight to more likely erroneous subcarriers. Therefore, the reference signal is also better. The improvement in additive white Gaussian noise case can be explained with the use of the cyclic prefix in the phase-noise estimation process.

\section{CONCLUSION}

Transmitter and receiver phase noises heavily affect the performance of an OFDM-based cognitive-radio link. This paper proposed a new received-side algorithm for joint transmitter and receiver phase-noise mitigation. The performance of the algorithm was compared to the performances of the state-of-the art algorithms. The proposed technique was seen to be able to give a clear performance improvement over the previous algorithms in the literature, when extended ITU-R Vehicular A multipath channel was assumed in the communications link. More generally, the results demonstrate that the signal distortion due to imperfect oscillators in multicarrier receivers can be efficiently suppressed. Such methods are seen essential in full deployment of dynamic spectrum access and cognitive radio, especially when the available and possible heavily scattered narrow spectral slices are used for secondary radio communications in the presence of strong neighbouring channels. Thus the DSP-enhanced RF hardware methods, like the one described in this article, are seen essential building blocks towards full-scale opportunistic spectrum access with practical RF circuits.

\section{REFERENCES}

[1] B. Wang and K. J. R. Liu, "Advances in cognitive radio networks: A survey," IEEE Journal on Selected Topics in Signal Processing, Vol. 5, No. 1, pp. 5-23, February 2011.

[2] M. Valkama, A. Springer, and G. Hueber, "Digital signal processing for reducing the effects of RF imperfections in radio devices - An overview," in Proc. International Symposium on Circuits and Systems (ISCAS'10), Paris, France, May-June 2010, pp. 813-816.

[3] G. Fettweis, "Dirty-RF: A new paradigm," in Proc. $16^{\text {th }}$ International Symposium on Personal, Indoor and Mobile Radio Communications 2005 (PIMRC'05), Berlin, Germany, September 2005, pp. 2347-2355.

[4] B. Razavi, "Cognitive radio design challenges and techniques," IEEE Journal on Solid-State Circuits, Vol. 45, pp. 1542-1553, August 2010.

[5] S. Wu, and Y. Bar-Ness, "OFDM systems in the presence of phase noise: consequences and solutions," IEEE Transactions on Communications, Vol. 52, No. 11, pp. 1988-1997, November 2004.

[6] D. Petrovic , W. Rave, and G. Fettweis, "Effects of phase noise on OFDM systems with and without PLL: characterization and compensation," IEEE Transactions on Communications, Vol. 55, No. 8, pp. 1607-1616, August 2007.

[7] S. Bittner, W. Rave, and G. Fettweis, "Joint iterative transmitter and receiver phase noise correction using soft information," in Proc. IEEE International Conference on Communications 2007 (ICC'07), Glasgow, Scotland, June 2007, pp. 2847-2852.

[8] S. Bittner, E. Zimmermann, and G. Fettweis, "Exploiting phase noise properties in the design of MIMO-OFDM receivers," in Proc. IEEE Wireless Communications and Networking Conference 2008 (WCNC'08), Las Vegas, NV, March 2008, pp. 940-945.

[9] V. Syrjälä, M. Valkama, N. N. Tchamov, and J. Rinne, "Phase noise modelling and mitigation techniques in OFDM communications systems," in Proc. Wireless Telecommunications Symposium 2009 (WTS'09), Prague, Czech Republic, April 2009.

[10] V. Syrjälä and M. Valkama, "Analysis and mitigation of phase noise and sampling jitter in OFDM radio receivers," International Journal of Microwave and Wireless Technologies, Vol. 2, No. 2, pp. 193-202, April 2010.

[11] V. Syrjälä and M. Valkama, "Receiver DSP for OFDM systems impaired by transmitter and receiver phase noise," in Proc. IEEE International Conference on Communications 2011 (ICC'11), Kyoto, Japan, June 2011.

[12] T. Schenk, RF Impairments in Multiple Antenna OFDM: Influence and Mitigation, $\mathrm{PhD}$ dissertation, Technische Universiteit Eindhoven, 2006. ISBN 90-386-1913-8. $291 \mathrm{p}$.

[13] A. Goldsmith, Wireless Communication, Cambridge University Press, 2005, 672 p. ISBN 978-0521837163.

[14] T. B. Sorensen, P. E. Mogersen, and F. Frederiksen, "Extension of the ITU channel models for wideband (OFDM) systems," in Proc. IEEE Vehicular Technology Conference 2005 (VTC'05-Fall), Dallas, TX, September 2005, pp. 392-396. 